\title{
Effects of Bifidobacterium lactis Bb12 Supplementation on Body Weight, Fecal pH, Acetate, Lactate, Calprotectin, and IgA in Preterm Infants
}

\author{
RUCHIKA MOHAN, CORINNA KOEBNICK, JANKO SCHILDT, MANFRED MUELLER, \\ MICHAEL RADKE, AND MICHAEL BLAUT
}

\begin{abstract}
Department of Gastrointestinal Microbiology [R.M., M.B.], German Institute of Human Nutrition Potsdam-Rehbruecke, Nuthetal 14558, Germany; Research and Evaluation [C.K.], Kaiser Permanente, Pasadena, California 91101; Klinik für Kinder und Jugendliche [J.S., M.M., M.R.], Klinikum Ernst von Bergmann, Potsdam 14467, Germany
\end{abstract}

\begin{abstract}
Preterm infants are prone to abnormal bacterial colonization of the intestine with ensuing adverse health effects. To examine whether the oral application of Bifidobacterium lactis Bb12 (probiotic) may improve selected indicators of health status in preterm infants, a double blind, placebo controlled randomized clinical study was performed on 69 preterm infants ( $<37$ gestation wk). Weight gain was defined as the primary outcome measure. In antibiotic-treated infants, probiotic supplementation resulted in a higher body weight compared with placebo $(p<0.001)$. In the probiotic group, the fecal $\mathrm{pH}$ was significantly lower than in the placebo group. The fecal concentrations of acetate and lactate were 42 and $38 \%$ higher, respectively, in the probiotic group than in the placebo group. Fecal calprotectin was lower in the probiotic group $(p=0.041)$, while fecal $\operatorname{IgA}$ was higher in this group compared with the placebo group ( $p=0.021)$. (Pediatr Res 64: 418-422, 2008)
\end{abstract}

$\mathrm{L}$ ow-birth-weight premature infants, in particular those delivered by caesarian section, are often ill equipped for life outside the womb. Because they spend the first few days of their life in an intensive care setting, they develop an abnormal pattern of microbial bowel colonization (1) and the proliferation of pathogens may lead to infections such as septicemia or necrotizing enterocolitis $(2,3)$. These infants are prone to infections because the mucosal barrier is poorly developed in low-birth-weight infants. The administration of probiotics has been proposed to reduce bowel infections in preterm infants by improving the mucosal barrier, thereby limiting the translocation of bacteria and bacterial products (4-6).

Previous studies indicated that probiotics might offer potential benefits to preterm and term infants (7-10). A recent review, which also included the first part of the present study, concluded that probiotics might reduce the risk of necrotising enterocolitis in preterm neonates with less than 33 wk gesta-

Received March 19, 2008; accepted May 14, 2008.

Correspondence: Michael Blaut, Ph.D., Department of Gastrointestinal Microbiology, German Institute of Human Nutrition Potsdam-Rehbruecke, Arthur-Scheunert-Allee 114-116, Nuthetal 14558, Germany; e-mail: blaut@dife.de

The authors did not have any financial or personal conflicts of interest. The study was supported by Nestlé who donated the food-grade study preparations and covered approximately $5 \%$ of the total study costs, the remainder being financed by the authors' institutions. Nestlé was not involved in the study design, the collection and interpretation of the data, writing of the report, and the decision to submit the paper for publication. None of the authors of the study received any payment from the sponsor. tion (11). Most of these studies looked at the effect of probiotics on microbial composition and weight gain as well as the occurrence of necrotizing enterocolitis, urinary tract infection, and sepsis. The present study investigated the effect of $\mathrm{Bi}$ fidobacterium lactis Bb12 supplementation on body weight, alteration of gut fermentation patterns, and immunologic parameters such as fecal calprotectin and IgA. The microbiological data from this study have already been published (12).

\section{METHODS}

Study design and study population. A double-blind placebo-controlled randomized prospective clinical trial was carried out on 69 preterm infants born between August 2003 and July 2005 at the Ernst von Bergmann hospital in Potsdam, Germany. The study was in agreement with the European "Good Clinical practice" and the protocol was approved by the Ethical committee of the University of Potsdam (decision number 3/15). Informed consent from the parents was obtained before enrollment of the infants. The study design has been described previously (12). In brief, infants born with a gestational age $<37$ wk were enrolled in the study and randomized into the placebo or the probiotic group with the help of the Randoma software version 4.3 (HaSoTec, Rostock, Germany) on the basis of birth weight, gestational age at birth, gender, arterial umbilical cord $\mathrm{pH}$, and Apgar score at 5 min. The physicians, nursing staff, parents, and the scientists analyzing the data were blinded to the treatment assignment. The placebo (human milk fortifier without probiotics: FM 2000B) and the probiotic preparation (FM 2000A) were both supplied by Nestlé, Konofingen, Switzerland. The probiotic supplement contained $2 \times$ $10^{9}$ cells of Bifidobacterium lactis Bb12 per g of powder, whereas the placebo was without $\mathrm{Bb} 12$. The probiotic group received oral feedings of $1.6 \times 10^{9}$ cells on d 1-3 and $4.8 \times 10^{9}$ cells from d 4 onwards daily. The first dose dissolved in water was given within $24 \mathrm{~h}$ after birth whenever possible. The infants received placebo or probiotic for $21 \mathrm{~d}$. The study preparation was given in addition to mother's milk or donated human milk. The dailyconsumed amounts of milk were noted.

Fecal samples were collected between 00:00 and 08:00 h for $21 \mathrm{~d}$ after the first supplementation. Because the quantity of stool samples was a limitation, not all parameters, except the fecal $\mathrm{pH}$, could be determined for each fecal sample. Stools collected on Mondays and Thursdays were processed for short chain fatty acids (SCFA) and lactate analysis, whereas those collected on Wednesdays were assayed for calprotectin and IgA. The samples for microbial analysis were collected on Tuesdays; the corresponding data have been reported previously (12). The number of subjects analyzed for a given parameter depended on the availability of a fecal sample at the respective sampling time; the number of subjects analyzed is indicated for each parameter.

Infants were subjected to standard antibiotic therapy used in the hospital when needed, which included cefotaxime $\left(200 \mathrm{mg} \mathrm{kg}-1 \cdot \mathrm{d}^{-1}\right)$ and piperacillin $\left(150 \mathrm{mg} \cdot \mathrm{kg}^{-1} \cdot \mathrm{d}^{-1}\right)$ in the first $3 \mathrm{~d}$, and vancomycin $\left(15 \cdot \mathrm{mg} \cdot \mathrm{kg}^{-1} \cdot \mathrm{d}^{-1}\right)$ and amikacin $\left(15 \cdot \mathrm{mg} \cdot \mathrm{kg}^{-1} \cdot \mathrm{d}^{-1}\right)$ after the third day.

Abbreviations: SCFA, short chain fatty acids 
Table 1. Study group characteristics. The probiotic and the placebo group contained 37 and 32 infants, respectively

\begin{tabular}{lcc}
\hline & Probiotic & Placebo \\
\hline Mode of delivery (\%) & & \\
$\quad$ Vaginal & 13.5 & 9.4 \\
$\quad$ Caesarian & 86.5 & 90.6 \\
Birth $(\%)$ & & \\
$\quad$ Single & 56.8 & 53.1 \\
$\quad$ Twins & 37.8 & 34.4 \\
$\quad$ Triplets & 5.4 & 12.5 \\
Mode of feeding $(\%)$ & & \\
$\quad$ Breast milk feeding (mother's milk or & 83.8 & 84.4 \\
$\quad$ donor milk) mostly supplemented & & \\
$\quad$ with fortifier & & \\
Formula feeding & 16.2 & 15.6 \\
Randomization parameters & & \\
$\quad$ Gender $(\%)(p=0.806)$ & 62.2 & 65.6 \\
$\quad$ Female & 37.8 & 34.4 \\
$\quad$ Male & $1449 \pm 343$ & $1398 \pm 331$ \\
Birth weight $(\mathrm{g})(p=0.947)$ & $7.31 \pm 0.07$ & $7.29 \pm 0.09$ \\
Arterial umbilical chord $\mathrm{pH}(p=0.165)$ & $31.05 \pm 2.31$ & $31.27 \pm 2.56$ \\
$\quad$ Gestation week $(p=0.575)$ & $8.03 \pm 1.50$ & $8.19 \pm 1.44$ \\
$\quad$ Apgar at 5 min $(p=0.698)$ & & \\
\hline
\end{tabular}

Imipenum $\left(60 \cdot \mathrm{mg} \cdot \mathrm{kg}^{-1} \cdot \mathrm{d}^{-1}\right)$ was given only in some cases. Baseline and the routine clinical data were collected for all infants and their mothers.

Fecal pH, short-chain fatty acids, and lactate. The fecal $\mathrm{pH}$ was measured directly on freshly passed stool with a Hamilton Biotrode glass electrode connected to a MV88 pH meter (both from Präcitronic, Dresden, Germany). SCFA were measured from $300 \mathrm{mg}$ fresh feces as described (13) with a HP 5890 series II gas chromatograph (Hewlett-Packard, Waldbronn, Germany). For lactate determination, the fecal sample was diluted 10-fold, heated to $80^{\circ} \mathrm{C}$ for $20 \mathrm{~min}$ to inactivate the enzymes and then finally centrifuged at $15,000 \times \mathrm{g}$ for $10 \mathrm{~min}$. The supernatant was analyzed for lactate enzymatically (Boehringer Mannhein/R-Biopharm, Darmstadt, Germany). The measurements were done in a microtiter plate and the absorption was read by a microtiter plate reader (Spectra RAINBOW, SLT Labinstruments, Austria)

Fecal calprotectin and IgA. Fecal calprotectin and $\operatorname{IgA}$ were measured with the PhiCal and the IgA ELISA kits (Immundiagnostik AG, Bensheim, Germany), respectively, using $100 \mathrm{mg}$ of fresh feces. The absorbance was measured at $405 \mathrm{~nm}$ with the above microtiter plate reader.

Data analysis. All statistical analyses were performed with the statistical package SPSS 11.5 (SPSS Inc., Chicago, IL). Sample size calculation was based on an assumed body weight difference between treatment groups of $100 \pm 140 \mathrm{~g}$. For this effect size, the power of the study was $86 \%$ with a sample size of 35 subjects per group and a given $\alpha=0.05$. Because the variables were not normally distributed, they were log transformed before performing any analysis. Geometric estimated means and standard errors are given in all figures. The influence of antibiotic therapy was also checked by dividing the probiotic and the placebo group infants into two groups: one without antibiotic therapy and the other in which the infants received antibiotic therapy for one or more days in the specified week. Antibiotic therapy was encoded on a weekly basis. Consequently, individual infants receiving antibiotic therapy changed from week to week.
Linear mixed effects modeling was used to estimate the effects of probiotic treatment on body weight. The hypothesis was that the difference between probiotic and placebo group changes over treatment time. For analysis, the data were adjusted for gestational week, birth weight, and gender. Age was centered at the end of treatment period (age $=3 \mathrm{wk}$ or $21 \mathrm{~d}$ ) for ease of interpretation of the intercept, because a zero age intercept would not be meaningful. The intercept represents the endpoint of the treatment period. For all models, fixed effects included were treatment (probiotic versus placebo) and antibiotic therapy. The effect was tested for significant fixed effects on the intercept and slope. A general mixed linear model was used for analyses with subject as a random factor and antibiotic therapy, treatment (probiotic or placebo), time of sample collection (week)*treatment, and antibiotic therapy*treatment as fixed factors for all other parameters (fecal pH, SCFA, lactate, calprotectin, and $\operatorname{IgA}$ ). Intrasubject correlation was calculated to estimate associations between fecal SCFA, IgA, calprotectin, lactate, and the numbers of bifidobacteria. The latter were reported elsewhere (12). The missing data at any particular time point was not taken for analysis. Weekly and overall effect of probiotic supplementation (considering data from all 3 wk) was calculated. The differences were considered significant at $p<0.05$ for all analyses.

\section{RESULTS}

Study group characteristics and effect of supplementation on weight gain. Of the 69 infants in the study, 37 and 32 infants were randomly assigned to the probiotic and the control group, respectively. The characteristics of the study population are shown in Table 1. All infants in the study were of German ethnic background, except one infant who was of Russian descent. During the study period of 3 wk, 46 infants received antibiotics (probiotic group: 26; placebo group: 20) for one or more weeks. Probiotic and control group did not differ with regard to the parameters chosen for randomization (Table 1). There were no significant differences in birth weight, arterial umbilical cord $\mathrm{pH}$ and Apgar score at $5 \mathrm{~min}$ between the infants born as singles, twins and triplets.

Probiotic treatment had no effect on weight when all infants were considered [placebo $1836 \pm 71$, probiotics $1882 \pm 53$, $p=0.062$; data given for $\mathrm{d} 21$ (time variable, centered at $\mathrm{d}$ $21)]$. When we investigated antibiotic treatment as a potential effect modifier, there was also no difference in weight between the probiotic and the control group for the infants without antibiotic treatment (placebo $1941 \pm 79$, probiotics $1900 \pm$ 78) (Fig. 1A). However, when the infants receiving antibiotics were compared, infants in the probiotic group had significantly higher weight than those in the placebo group [placebo $1375 \pm 74$, probiotics $1574 \pm 65, p<0.001$ for $\mathrm{d} 21$ (time variable, centered at d 21) $\times$ antibiotic $\times$ treatment] (Fig. $1 B$ ). Interestingly, the milk intake by the infants in the placebo group was significantly higher than by the infants in the
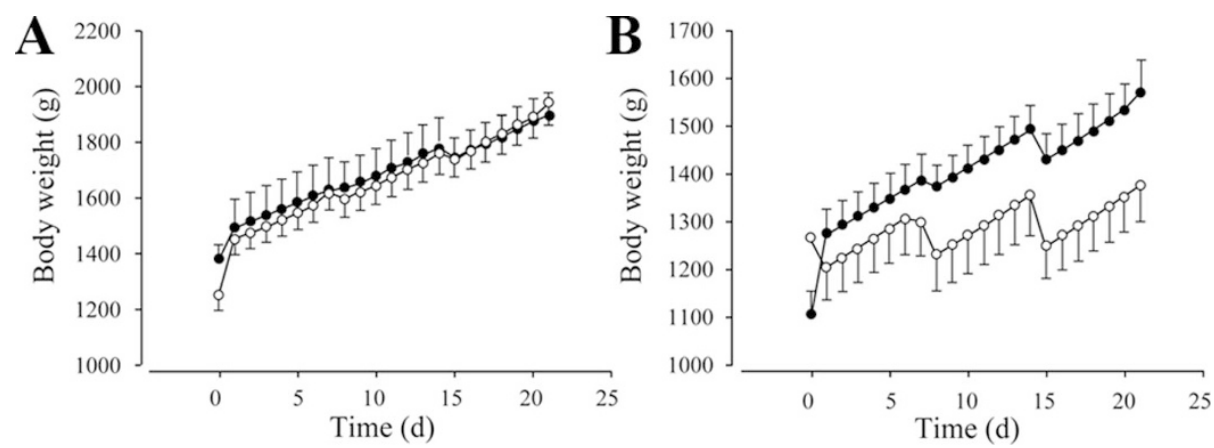

Figure 1. Influence of probiotic treatment on body weight. $(A)$ Infants not treated with antibiotics ( $p=$ NS; probiotic: $n=11$, placebo $=$ $12),(B)$ antibiotic-treated infants $(p<0.001$; probiotic: $n=26$, placebo $=20$ ). Placebo group $(\bigcirc)$; probiotic group $(\bullet)$. 

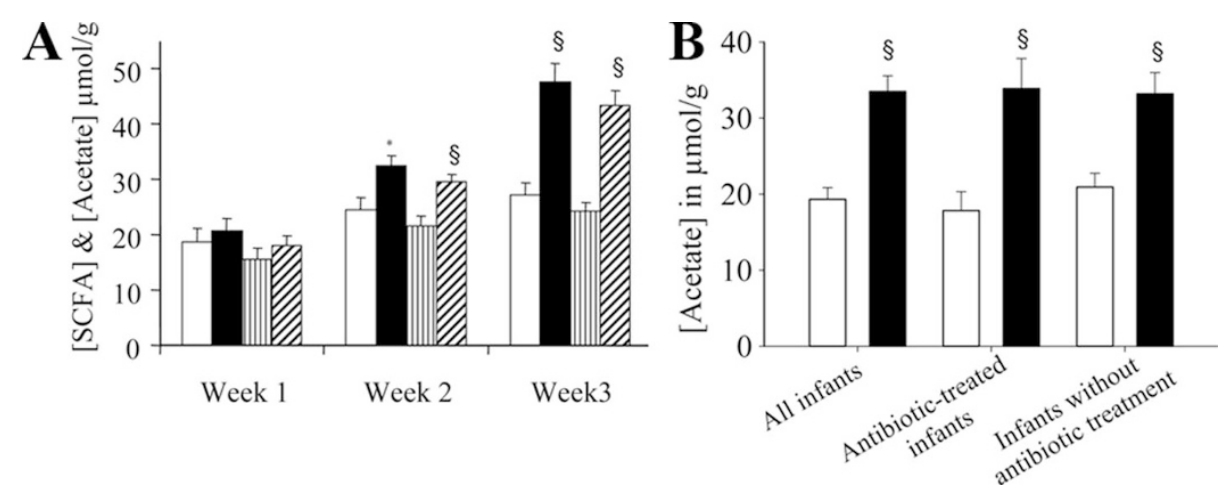

Figure 2. (A) Effect of probiotic supplementation on fecal SCFA and acetate concentrations over a period of three study weeks (probiotic: $n=34$, placebo $=$ 31) given as geometric means. $\square$ : total SCFA in the placebo group; $\mathbf{\square}$ : total SCFA in the probiotic group; 四: acetate in the placebo group; 四: acetate in the probiotic group. $(B)$ Effect of probiotic supplementation on fecal acetate concentration in all infants $(p<0.001$; probiotic: $n=31$, placebo $=28)$, antibiotic-treated infants $(p<0.001$; probiotic: $n=23$, placebo $=19)$, and in infants without antibiotic treatment $(p<0.001$; probiotic: $n=11$, placebo $=$ 12) over the entire study period (3 wk). Placebo group ( $\square$ ); probiotic group ( $\square$ ). ${ }^{*} p<0.05$; $\$ p<0.001$.

probiotic group (placebo $226 \mathrm{ml} / \mathrm{wk} \pm 5.25$; probiotic 199 $\mathrm{ml} / \mathrm{wk} \pm 4.16, p<0.001)$.

Fecal SCFA, lactate, and $\mathrm{pH}$. The total concentrations of fecal SCFA in the probiotic $(n=34)$ and placebo $(n=31)$ groups are shown in Figure 2A. Considering the data from all time points, the probiotic group had a higher total fecal SCFA concentration $(p<0.001)$ than the placebo group and the differences were most pronounced in wk 2 and 3 ( $p=0.013$ and $p<0.001$, respectively). Acetic acid was the major SCFA in both groups, contributing $90 \%$ to the total SCFA. The concentration of acetate was significantly higher in the probiotic than in the placebo group $(p<0.001)$ with more pronounced differences in the second and third weeks (Fig. 2A, $p<0.001$ for both weeks). The differences between the two groups were also significant for the infants who received antibiotics $(p<0.001$, Fig. $2 B)$. Fecal propionic and butyric acid concentrations were consistently higher in the probiotic than in the placebo group $(p=0.040 ; p=0.026$, respectively). The geometric mean of the molar proportions of SCFA profile for all time points was similar for the probiotic and the placebo group $\left(\mathrm{C}_{2}: \mathrm{C}_{3}: \mathrm{C}_{4}: \mathrm{C}_{5}\right.$; probiotic: $90: 4: 3.2: 2$; placebo: 89.3:5:3:2).

Lactate was produced by the intestinal microbiota of all infants in both study groups (probiotic, $n=35$; placebo, $n=$ 31). Fecal lactate increased with age in both groups (not shown), but the infants in the probiotic group had a $38 \%$ higher fecal lactate concentration $(p=0.011)$ (Fig. 3). The differences between the two groups were more pronounced in the infants without antibiotic therapy $(p=0.009)$. In the infants receiving antibiotics, the fecal lactate concentrations were not significantly different between the probiotic and the placebo group.

Bifidobacterial numbers, presented elsewhere (12), correlated significantly with fecal acetate and lactate $(r=0.243$, $p=0.050 ; r=0.336, p=0.010$, respectively). A significant correlation was also found for fecal acetate and lactate $(r=$ $0.490, p<0.001)$. The feces from infants in the probiotic group $(5.68 \pm 0.09)$ had a significantly lower $\mathrm{pH}(p<0.001)$ than those in the placebo group $(6.38 \pm 0.10)$.

Fecal calprotectin and fecal IgA. The fecal calprotectin levels were significantly higher $(p=0.041)$ in the placebo

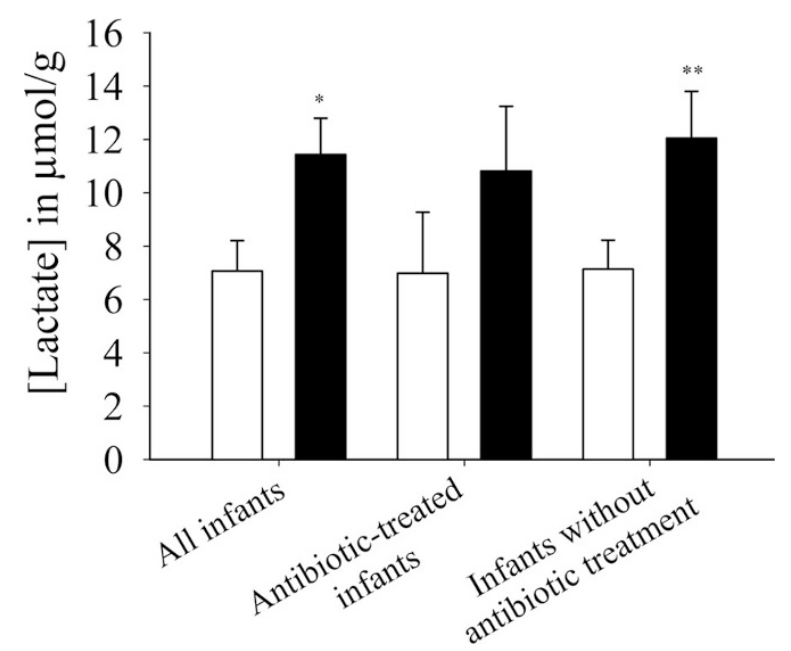

Figure 3. Effect of probiotic supplementation on fecal lactate concentration (in micromole per gram feces) in all infants ( $p=0.011$; probiotic: $n=35$, placebo $=31$ ), antibiotic-treated infants (probiotic: $n=24$, placebo $=20$ ), and in infants without antibiotic treatment ( $p=0.009$; probiotic: $n=10$, placebo $=12$ ) over the entire study period of 3 wk. Placebo group $(\square)$; Probiotic group (ם). $* p<0.050 ; * * p<0.010$.

group than in the probiotic group considering all data points. Although the difference between the two groups was not significant for the infants receiving antibiotics, it was significant for the infants without antibiotic treatment $(p=0.007)$ (Fig. 4).

Total fecal IgA levels were $44 \%$ higher in the probiotic $(n=19)$ than in the placebo group $(n=16 ; p=0.021)$. The fecal IgA concentration increased from wk 1-2 in the probiotic group, but not in the placebo group (Fig. 5A). However, this difference was only significant for infants without antibiotic treatment (Fig. 5B). A trend for a positive correlation of fecal IgA with the cell counts of bifidobacteria (12), was observed $(r=0.344, p=0.080)$.

\section{DISCUSSION}

It is known that very low-birth-weight infants lose up to $10 \%$ of their initial weight during the immediate postnatal period (6). This creates a significant growth deficit, which 


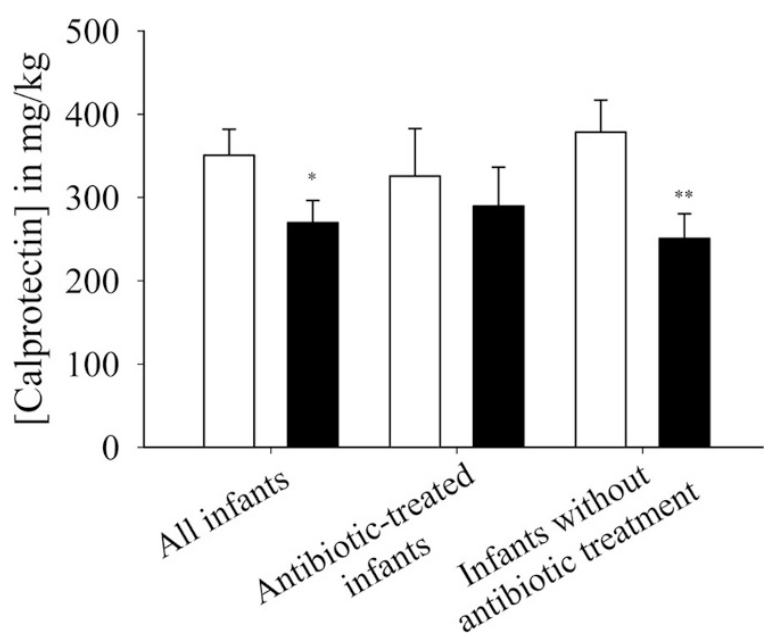

Figure 4. Effect of probiotic supplementation on fecal calprotectin concentration (in milligram per kilogram feces) in all infants ( $p=0.041$; probiotic: $n=34$, placebo $=29$ ), antibiotic-treated infants (probiotic: $n=24$, placebo $=18)$, and in infants without antibiotic treatment $(p=0.007$; probiotic: $n=10$, placebo $=11$ ) over the entire study period ( $3 \mathrm{wk}$ ). Placebo group $(\square)$; probiotic group (ם). $* p<0.050 ; * * p<0.010$.

persists throughout hospitalization in a neonatal intensive care unit. In the present study, antibiotic-treated infants who received probiotics gained more weight than those supplemented with placebo, although the infants in the probiotic group consumed smaller amounts of milk. Indeed, the weight gain during the observation period was four times higher for the infants in the probiotic group than for the infants in the placebo group (approximately $450 \mathrm{~g}$ versus $100 \mathrm{~g}$; Fig. 1B). It is not completely clear why BB12 treatment only affected the weight of infants who were treated with antibiotics. However, this finding may indicate that probiotic treatment is advantageous in infants suffering from bacterial infections and requiring antibiotic treatment. Because the infants in the antibiotic-treated group were already ill, the probiotics showed a higher effect in these infants. It can be proposed that either the infants in the probiotic group lost less of their initial weight than the infants in the placebo group or they digested and used the food more effectively than the ones in the latter group. Improved weight gain in preterm infants on supplementation with Bifidobacterium breve YIT4010 has also been shown previously (7).

The infants without antibiotic treatment had a higher body weight at the end of the observation period (approximately
$1900 \mathrm{~g}$ for both the probiotic and the placebo group) than those with antibiotic treatment (approximately $1370 \mathrm{~g}$ for the placebo and approximately $1580 \mathrm{~g}$ for the probiotic group). This can be explained by the fact that the infants with antibiotic treatment had a lower birth weight than those without it, which may indicate that a low-birth-weight predisposes preterm infants to the need for antibiotic treatment.

In the first few days of life, the large bowel contains no or very few bacteria that degrade dietary products or endogenous glycoproteins. Moreover, the availability of exogenous substrates for intestinal bacteria is limited because of the low food intake during this period. Therefore, the production and absorption of SCFA are unlikely to occur at significant levels until the infant acquires an anaerobic microbiota (14) and milk uptake increases. Fecal SCFA reflect bacterial fermentation of carbohydrates and proteins of exogenous and endogenous origin. Very little is known about the fecal SCFA concentrations in premature infants, especially in relation to digestive disorders and probiotic supplementation.

Bifidobacteria produce acetate and lactate at a molar ratio of 3:2. The total fecal SCFA concentrations in the probiotic group were higher than those in the placebo group, with acetate accounting for approximately $90 \%$ of the total SCFA. Other studies on premature infants reported molar acetic acid proportions of $65 \%$ (15) and 69\% (16). So far, no previous study has monitored the fecal lactate levels after supplementation of probiotics in preterm infants. The fecal concentration of lactate is less than $2-3 \mathrm{mM}$ in adults (17) but considerably higher in infants. This can be attributed to a lack of lactatedegrading bacteria in infants. In the present study, the concentration of lactic acid in the feces increased almost 2.6-fold from wk 1 to 3 in the infants supplemented with B. lactis $\mathrm{Bb} 12$. This can be linked to the observed increase in the number of bifidobacteria during this time period (12) and is evident from the significant positive correlation between the two parameters. Improved lactose digestion has been reported for humans by ingestion of unfermented milk containing Bifidobacterium longum (18). Because, human milk contains relatively high concentrations of lactose, bifidobacteria have an advantage over other bacteria that do not possess $\beta$-galactosidase. The lower fecal $\mathrm{pH}$ detected in the infants of the probiotic group can be explained by the higher amounts of fecal acetic and lactic acids found in these infants.
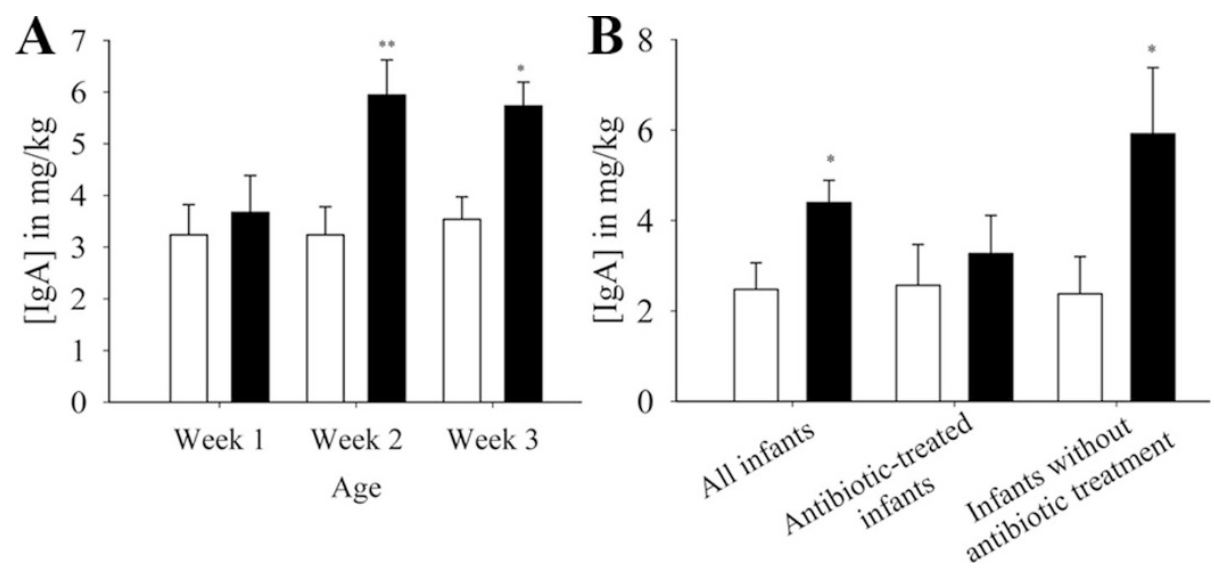

Figure 5. (A) Effect of probiotic supplementation on fecal $\operatorname{IgA}$ concentrations (in milligram per kilogram feces) of preterm infants over 3 wk. (B) Effect of probiotic supplementation on fecal IgA concentrations in all infants $(p=$ 0.021; probiotic: $n=19$, placebo $=16$ ), antibiotic-treated infants (probiotic: $n=13$, placebo $=13)$, and in infants without antibiotic treatment ( $p=0.018$; probiotic: $n=6$, placebo $=3$ ) over the entire study period $(3 \mathrm{wk})$. Placebo group ( $\square$ ); probiotic group (ם). $* p<$ $0.050 ; * p<0.010$. 
Calprotectin is a $36.5 \mathrm{kD}$ calcium and zinc binding protein, which constitutes approximately $60 \%$ of the soluble cytosolic proteins in the human neutrophil granulocytes (19). Elevated concentrations of fecal calprotectin are found in patients with colorectal cancer, inflammatory bowel disease, bacterial infections of the gastrointestinal tract and after treatment with nonsteroidal antiinflammatory drugs (20-22). It is a simple noninvasive marker to evaluate the inflammatory status of the gastrointestinal tract of neonates, particularly of those at risk of necrotizing enterocolitis, preterm infants, and infants with intrauterine growth retardation (23). Few studies have looked at the fecal calprotectin levels in preterm infants (24-26), but none of them examined the effect of probiotic supplementation. In the present study, the infants in the probiotic group had a significantly lower concentration of fecal calprotectin than those in the placebo group. Bb12 supplementation might help in mucosal immunity maturation and attenuation of inflammatory responses to dietary and bacterial antigens.

Secretory IgA plays a central role in local immunity. It has a significant function in creating a barrier against infections by pathogenic bacteria or viruses (27). An increase in total fecal IgA (2.9-fold) in healthy children in response to supplementation with $B$. lactis $\mathrm{Bb} 12$ has been shown previously (28). We observed a $45 \%$ increase in the total fecal IgA concentration from wk 1 to 2 in the probiotic group whereas there was no change in the concentration during the entire study period of $3 \mathrm{wk}$ in the placebo group.

In conclusion, dietary supplementation of preterm infants with $B$. lactis Bb12 starting early after birth led to an increase in fecal acetate, lactate, and total fecal IgA and to a decrease in fecal calprotectin. A significantly higher body weight in response to probiotic supplementation was only observed in infants treated with antibiotics.

\section{REFERENCES}

1. Bennet R, Eriksson M, Nord CE, Zetterstrom R 1986 Fecal bacterial microflora of newborn infants during intensive care management and treatment with five antibiotic regimens. Pediatr Infect Dis 5:533-539

2. La Gamma EF, Drusin LM, Mackles AW, Machalek S, Auld PA 1983 Neonatal infections. An important determinant of late NICU mortality in infants less than 1,000 g at birth. Am J Dis Child 137:838-841

3. Freedman RM, Ingram DL, Gross I, Ehrenkranz RA, Warshaw JB, Baltimore RS 1981 A half century of neonatal sepsis at Yale: 1928 to 1978. Am J Dis Child 135:140-144

4. Gupta P, Andrew H, Kirschner BS, Guandalini S 2000 Is Lactobacillus GG helpful in children with Crohn's disease? Results of a preliminary, open-label study. J Pediatr Gastroenterol Nutr 31:453-457

5. Salminen S, Isolauri E, Salminen E 1996 Clinical uses of probiotics for stabilizing the gut mucosal barrier: successful strains and future challenges. Antonie Van Leeuwenhoek 70:347-358
6. Walker WA 2000 Role of nutrients and bacterial colonization in the development of intestinal host defense. J Pediatr Gastroenterol Nutr 30:S2-S7

7. Kitajima H, Sumida Y, Tanaka R 1997 Early administration of Bifidobacterium breve to preterm infants: randomised controlled trial. Arch Dis Child Fetal Neonatal Ed 76:F101-F107

8. Bin-Nun A, Bromiker R, Wilschanski M, Kaplan M, Rudensky B, Caplan M, Hammerman C 2005 Oral probiotics prevent necrotizing enterocolitis in very low birth weight neonates. J Pediatr 147:192-196

9. Lin HC, Su BH, Chen AC, Lin TW, Tsai CH, Yeh TF, Oh W 2005 Oral probiotics reduce the incidence and severity of necrotizing enterocolitis in very low birth weight infants. Pediatrics 115:1-4

10. Szajewska H, Setty M, Mrukowicz J, Guandalini S 2006 Probiotics in gastrointestinal diseases in children: hard and not-so-hard evidence of efficacy. J Pediatr Gastroenterol Nutr 42:454-475

11. Deshpande G, Rao S, Patole S 2007 Probiotics for prevention of necrotizing enterocolitis in preterm neonates with very low birth weight: a systematic review of randomized controlled trials. Lancet 369:1614-1620

12. Mohan R, Koebnick C, Schildt J, Schmidt S, Possner M, Radke M, Blaut M 2006 Effects of Bifidobacterium lactis Bb12 supplementation on intestinal microbiota of preterm infants: a double-blind, placebo-controlled, randomized study. J Clin Microbiol 44:4025-4031

13. Schneider H, Schwiertz A, Collins MD, Blaut M 1999 Anaerobic transformation of quercetin-3-glucoside by bacteria from the human intestinal tract. Arch Microbiol 171:81-91

14. Walker V, Mills GA, Hall MA, Lowes JA 1989 Carbohydrate fermentation by gut microflora in preterm neonates. Arch Dis Child 64:1367-1373

15. Favre A, Szylit O, Popot F, Catala I, Rondeau C, Maurage C, Gold F, Borderon JC, Butel MJ 2002 Diet, length of gestation, and fecal short chain fatty acids in healthy premature neonates. JPEN J Parenter Enteral Nutr 26:51-56

16. Stansbridge EM, Walker V, Hall MA, Smith SL, Millar MR, Bacon C, Chen S 1993 Effects of feeding premature infants with Lactobacillus GG on gut fermentation. Arch Dis Child 69:488-492

17. Mortensen PB, Hove H, Clausen MR, Holtug K 1991 Fermentation to short-chain fatty acids and lactate in human faecal batch cultures. Intra- and inter-individual variations versus variations caused by changes in fermented saccharides. Scand J Gastroenterol 26:1285-1294

18. Jiang T, Mustapha A, Savaiano DA 1996 Improvement of lactose digestion in humans by ingestion of unfermented milk containing Bifidobacterium longum. J Dairy Sci 79:750-757

19. Fagerhol MK, Andersson KB, Narss-Andresen CF 1990 Calprotectin (the L1 leukocyte protein). In: Smith VL, Dedman JR (eds) Stimulus Response Coupling: the Role of Intracellular Calcium-Binding Proteins. CRC Press, Boca Raton, FL, pp $187-210$

20. Kristinsson J, Roseth A, Fagerhol MK, Aadland E, Schjonsby H, Bormer OP, Raknerud N, Nygaard K 1998 Fecal calprotectin concentration in patients with colorectal carcinoma. Dis Colon Rectum 41:316-321

21. Berstad A, Arslan G, Folvik G 2000 Relationship between intestinal permeability and calprotectin concentration in gut lavage fluid. Scand J Gastroenterol 35:64-69

22. Meling TR, Aabakken L, Roseth A, Osnes M 1996 Faecal calprotectin shedding after short-term treatment with non-steroidal anti-inflammatory drugs. Scand J Gastroenterol 31:339-344

23. Carroll D, Corfield A, Spicer R, Cairns P 2003 Faecal calprotectin concentrations and diagnosis of necrotising enterocolitis. Lancet 361:310-311

24. Campeotto F, Kalach N, Lapillonne A, Butel MJ, Dupont C, Kapel N 2007 Time course of faecal calprotectin in preterm newborns during the first month of life. Acta Paediatr 96:1531-1533

25. Josefsson S, Bunn SK, Domellof M 2007 Fecal calprotectin in very low birth weight infants. J Pediatr Gastroenterol Nutr 44:407-413

26. Nissen AC, Van Gils CE, Menheere PP, Van den Neucher AM, van der Hoeven MA, Forget PP 2004 Fecal calprotectin in healthy term and preterm infants. J Pediatr Gastroenterol Nutr 38:107-108

27. Underdown BJ, Schiff JM 1986 Immunoglobulin A: strategic defense initiative at the mucosal surface. Annu Rev Immunol 4:389-417

28. Fukushima Y, Kawata Y, Hara H, Terada A, Mitsuoka T 1998 Effect of a probiotic formula on intestinal immunoglobulin A production in healthy children. Int J Food Microbiol 42:39-44 